\title{
Drawing Liquid Metal Wires at Room Temperature
}

Yiliang Lin ${ }^{+}$, Collin Ladd ${ }^{+}$, Siyao Wang, Andre Martin, Jan Genzer, Saad A. Khan, Michael D. Dickey*

Department of Chemical \& Biomolecular Engineering, North Carolina State University, Raleigh, NC 27695-7905, USA

Keywords: liquid metal, room temperature fabrication, metallic wires

+ Y.L. and C.L. contributed equally to this work

*Corresponding authors. Email: mddickey@ncsu.edu

\section{TOC Graphic:}
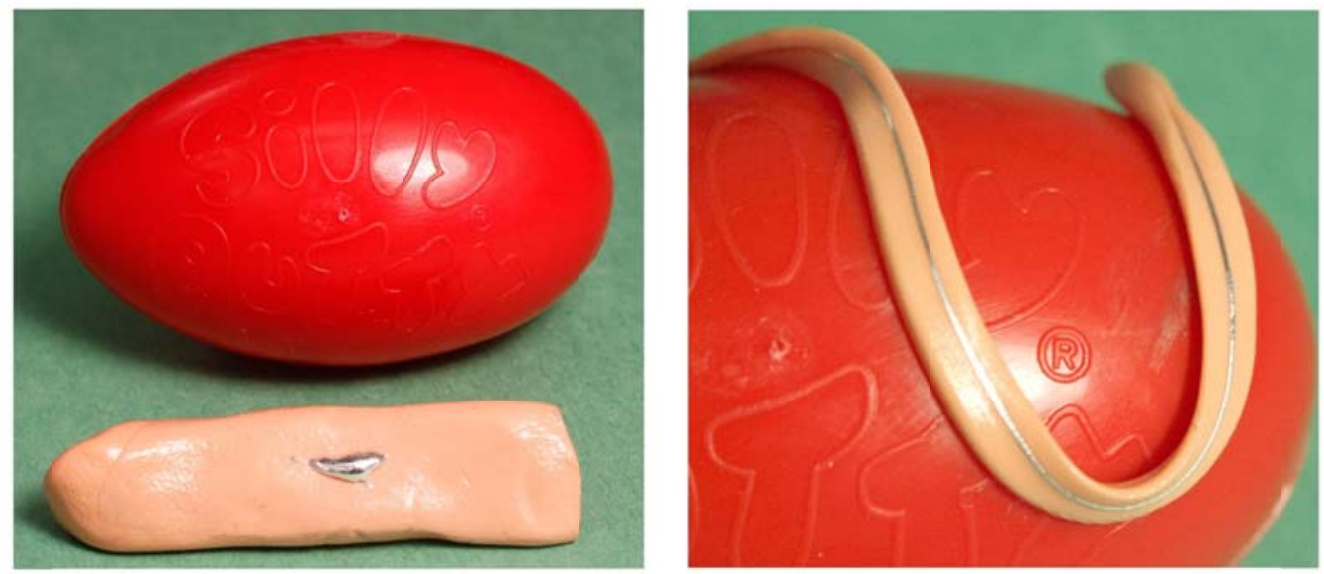


\begin{abstract}
This paper describes an extremely facile method to fabricate metallic wires at room temperature. The wires form by stretching viscoelastic polymer substrates supporting a drop of gallium-based liquid metal. Stretching the polymer causes the metal to also elongate due to the adhesion between the two materials. The diameters of the resulting wires, which can be as small as $10 \mu \mathrm{m}$, decrease with increasing strain. This method is inspired by the process used for drawing optical fibers, which involves pulling a pre-form cylinder of molten glass until it thins to the size of a fiber. In contrast, the process here is done at room temperature and realized without the need for large forces. Moreover, geometries beyond simple wires are possible including parallel, core-shell, branched, and helix structures. The resulting wires can be elastic (stretchable), viscoelastic (soft), or plastic (stiff) depending on the chemistry and post-processing of the polymer. Wires can make electrical contacts by allowing the metal to sink through the viscoelastic polymer onto a substrate containing electrodes. In addition, removing the polymer substrate after elongation produces freestanding liquid metal wires stabilized by the surface oxide on the metal. Rheological studies show that polymers with a variety of properties can be utilized to form these wires including viscoelastic materials and gels. The ability to form metallic wires in a simple manner may find uses in soft and stretchable electronics, or enable new applications, such as 'wires on demand' for repairing electrical connections.
\end{abstract}




\section{Introduction}

This paper describes an extremely simple method to fabricate metallic wires at room temperature with a wide range of mechanical properties. The wires form by manually stretching a polymer slab supporting a droplet of gallium-based liquid metal. Both the liquid metal and polymer substrate elongate during stretching to form wires in a process similar to drawing optical fibers (i.e., pulling a pre-form cylinder of glass softened by heat ${ }^{1}$ ). Others have taken inspiration from this high temperature process to elongate metals ${ }^{2}$, optical materials ${ }^{3}$, and semiconductors ${ }^{4}$ to make nanostructures ${ }^{4}$, probes $^{5}$, and sensors ${ }^{3}$. Here, we show it is possible to form metallic wires by hand at room temperature by utilizing materials that are inherently deformable and soft. Utilizing such a simple strategy forms conductive, metallic wires with diameters as small as tens of microns, embedded core-shell structures, parallel networks of wires, branched structures, and helices. Moreover, depending on the chemistry of the polymer substrates, the resulting metallic wires may be stretchable, rigid or soft by crosslinking the polymer after formation of the wires.

The fabrication of conventional electrical wires begins typically with a spool of metal 'rod' (e.g. copper) formed via high temperature processing. A series of pulleys and dies elongate the rod under tension using large forces until the diameter reduces to a suitable value; elevated temperatures can reduce the required forces ${ }^{6}$. The wires are often braided and then coated with plastic insulation via extrusion of polymer at elevated temperatures. The resulting wires are stiff due to the mechanical properties of the metal. We sought a simpler process to make insulated wires on demand, at room temperature, with a wide range of mechanical properties. The goal is not to replace conventional cabling, but rather provide an alternative method to make conductive traces that takes advantage of the unique properties of liquid metal.

Metals that are liquid at room temperature have two features that are appealing within the context of making wires: (1) they can be mechanically manipulated at room temperature, and (2) the resulting wires maintain metallic conductivity even at large deformation and are therefore useful for soft and stretchable electronics. ${ }^{7,8}$ Here, we utilize eutectic gallium indium (EGaIn) due to its low melting point (m.p. $\sim 15.5^{\circ} \mathrm{C}$ ) 9,10 , 
low-toxicity ${ }^{11}$ and high conductivity $\left(\sigma=3.4 \times 10^{4} \mathrm{~S} / \mathrm{cm}\right)^{12}$. Various microfluidic systems and soft electronics utilize this liquid metal ${ }^{13}$, such as soft memristors ${ }^{14}$, stretchable wires ${ }^{7,8}$, antennas ${ }^{15-19}$, electrodes ${ }^{20,21}$, pumps $^{22}$, capacitors ${ }^{23,24}$, soft circuit boards $^{25}$, hyperelastic pressure sensors $^{26}$, self-healing circuits $^{27,28}$, soft curvature sensors $^{29}$, and stretchable interconnects ${ }^{30,31}$. EGaIn forms a surface oxide that is important for two aspects of this work: (1) It allows the metal to adhere to the polymer during elongation, and (2) it mechanically stabilizes the metal to maintain high aspect ratio geometries that would be prohibited by surface tension due to Plateau-Rayleigh instabilities.

Various processes have been introduced for patterning liquid metals in polymers ${ }^{32}$, such as stencil lithography ${ }^{33,34}$, imprint lithography ${ }^{35}$, injection ${ }^{10,15}$, microcontact printing $^{36}$ and direct-writing ${ }^{37}$. Most existing patterning techniques are relatively low resolution $(>100 \mu \mathrm{m})$. Patterning at higher resolution may enable new applications such as dense wire bundles or electrodes with length scales commiserate with cells. The method here is distinguished by its simplicity and ability to make both metallic structures with small diameters and high aspect ratios. Metallic structures with high aspect ratios are desirable for conductive fibers, wires, interconnects, and cables. It is possible to make conductive fibers of liquid metal by melt processing or electrospinning hollow fibers filled with metal ${ }^{7,38}$, although these processes require tools that are not widely available. It is also possible to create conductive fibers utilizing conductive particles ${ }^{39}$ (e.g. $\mathrm{Ag}_{\text {particles }}{ }^{40}$ and $\mathrm{CNTs}^{41}$ ), although no other material offers the combination of conductivity and elasticity provided by liquid metals.

Here, we demonstrate that it is possible to draw either exposed or encapsulated wires of EGaIn at room temperature in a one-step process without any specialized equipment. The simple stretching approach can achieve liquid metal structures with diameters smaller than the resolution of many existing techniques to pattern liquid metal lines. Rheological and mechanical measurements confirm the properties of both the starting materials and resulting wires, which helps elucidate the mechanism of wire formation. 


\section{Result and Discussion}

Figure 1 highlights a simple approach for making metallic wires at room temperature. First, a syringe extrudes EGaIn on a polymer substrate (in this representative example, $\sim 50$ microliters of metal on $\sim 10 \mathrm{~g}$ of Silly Putty ${ }^{\mathrm{TM}}$ ). Stretching the putty by hand (Figure 1b) extends both the polymer and the metal, resulting in an elongated structure with a diameter that decreases with strain (Figure 1c). Supporting information (SI) Video S1 shows a wire being stretched. Using typical strain rates of $\sim 1 \mathrm{~cm} / \mathrm{s}$, EGaIn wires with diameters approaching the tens of micron-scale form within seconds using this strategy. Due to the soft and stretchable property of putty, conductive, metallic wires can be manipulated into a variety of geometries. Figure 1d provides an example of one such shape.
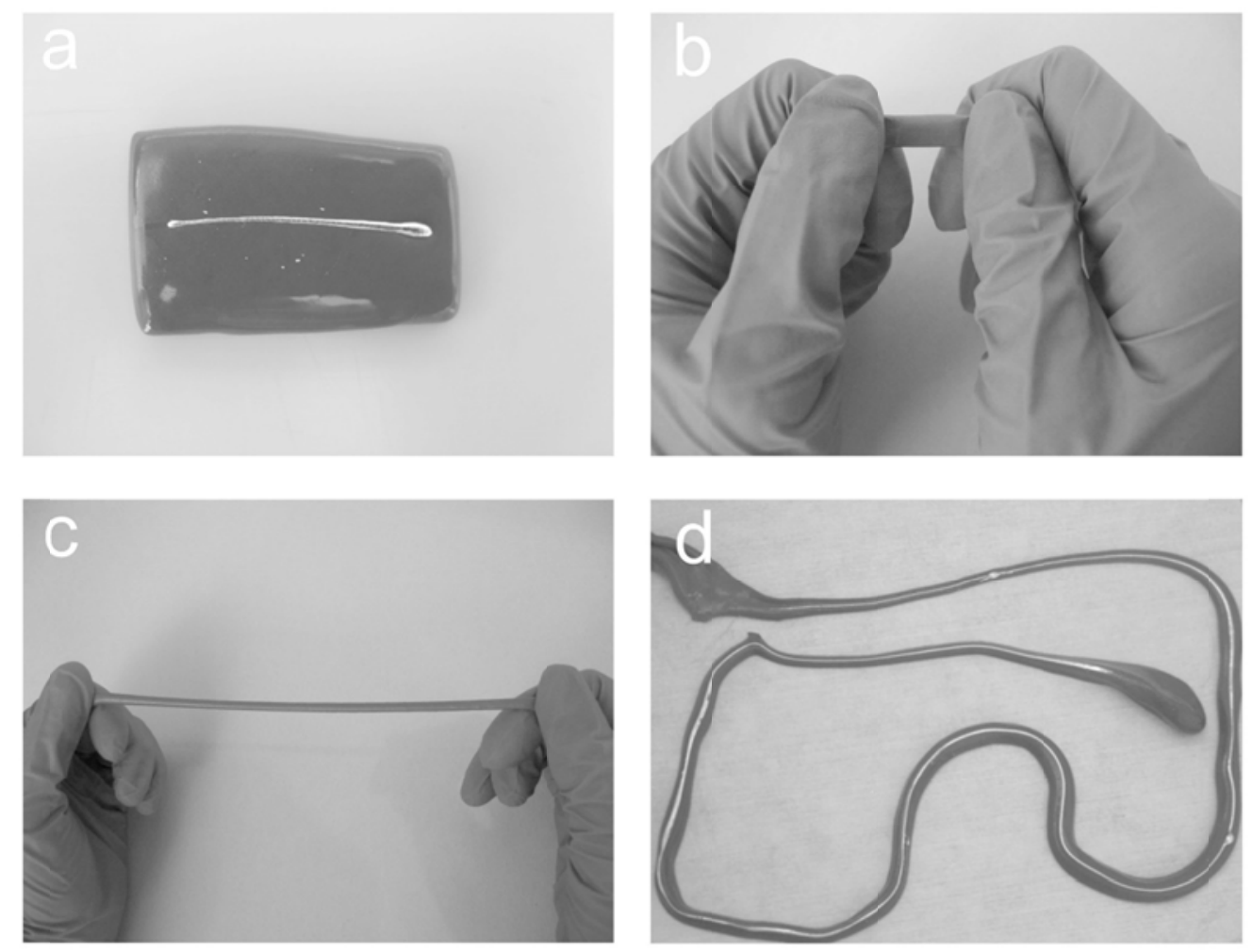

Figure 1. Drawing of EGaIn wires by hand at room temperature. (a) EGaIn lines dispensed on a putty (viscoelastic polymer) substrate. (b) Putty before stretching by hand. (c) Stretched putty with elongated liquid metal lines. (d) A curvy EGaIn wire supported by putty after stretching. 
We hypothesized that if the metal adheres well to the putty, the diameter should correlate with the strain of the polymer. Figure 2a plots the diameters of the wires as a function of the strains on the polymer substrate (Experimental Section in SI). Approximating the geometry of the EGaIn lines as cylinders and accounting for conservation of mass, results in Equation 1,

$$
\mathrm{V}=\frac{1}{4} \pi d_{0}^{2} l_{0}=\frac{1}{4} \pi d_{1}^{2} l_{1}=\frac{1}{4} \pi d_{1}^{2} n l_{0}
$$

which simplifies to Equation 2,

$$
d_{1}=d_{0} n^{-\frac{1}{2}}
$$

where $\mathrm{V}$ is the volume of the metal, $\mathrm{d}_{0}$ and $\mathrm{d}_{1}$ is the diameter of the EGaIn lines before and after stretching, and $l_{0}$ and $l_{1}$ is the length of EGaIn lines before and after stretching. We define $n$ as the stretch ratio $\left(l_{1} / l_{0}\right)$ of the EGaIn lines. Equation 2 also holds for truncated cylinders. As predicted by Equation 2, the diameters of the EGaIn lines measured experimentally vary linearly with the inverse square root of the stretch ratio, as shown in Figure 2b. Under an optical microscope, shiny liquid metal lines are evident against the darker putty substrate, as evident in Figure 2c. Figure 2d verifies that the wires are electrically continuous. Typically, the wires break when stretched to diameters below $10 \mu \mathrm{m}$, which may be due to the challenges associated with manipulating materials by hand at such a small scale or due to instabilities. In our experiments, liquid metal wires break before the putty substrate becomes discontinuous; it is possible to continue to stretch the polymer by hand to diameters as narrow as $2 \mu \mathrm{m}$ before failure. The measurements reported in Figure 2 suggest three important things: (1) It is possible to control the diameter of the wires with strain, (2) the oxide skin adheres EGaIn to the putty, and (3) the wires are electrically continuous. In regards to the second claim, we note that acid vapor (e.g. $\mathrm{HCl}$ ) removes the oxide and causes the bare metal to dewet from the surface of the putty, which confirms the importance of the oxide. Fresh oxide that forms during elongation of the metal should favor wetting according to prior studies on siloxanes ${ }^{42}$. 

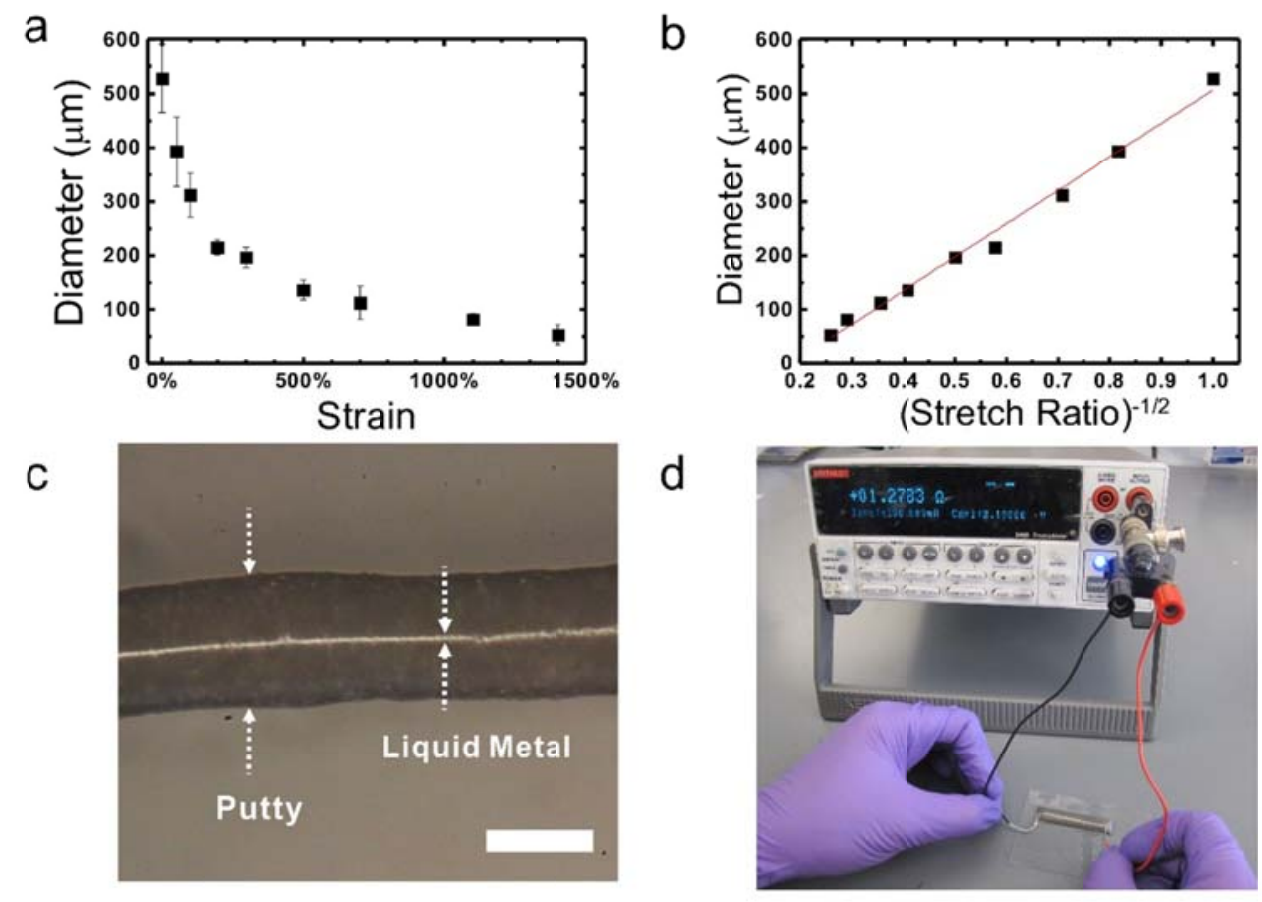

Figure 2. Characterization of the EGaIn wires on putty. (a) Plot for the diameters of EGaIn wires as a function of the strain applied to the putty. (b) Linear plot of diameter as a function of the inverse square root of the stretch ratio. The fit quality is $\mathrm{R}^{2}=0.98$. (c) Optical microscopic images of a small EGaIn wire. The scale bar is $50 \mu \mathrm{m}$. (d) The wires are electrically conductive.

Although stretching liquid metal is a simple method to form wires, there are at least two practical issues: (1) the wires are not encased completely in insulation, and (2) the putty can continue to flow after forming the wires. Addressing the first issue is straightforward. Figure 3a shows that encapsulated wires form by first injecting a drop of the metal into a slab of the putty and then drawing it into a wire. The second issue (which we show later to be advantageous in certain scenarios) can be addressed by utilizing polymers that can be chemically modified (e.g. crosslinked) during post-processing to form both elastomeric and stiff wires. To form elastomeric wires, we first encased metal into a slab of poly(dimethylsiloxane) (Dow Corning SE 1700) and drew it into a wire, as shown in Figure 3b. We chose SE 1700 mainly due to its 
ease of processing, strong elasticity after curing, and high elongation value $(>350 \%$ strain). The resulting core-shell (i.e., insulated) wires are stretchable, twistable and bendable and recover the original shape after deformation. In addition, Figure 3c shows it is also possible to make wires encased in a stiff matrix by drawing the liquid metal in a polyvinyl alcohol (PVA) matrix containing an aqueous solution of borax. (Experimental Section 2 in SI) The water evaporates and the borax slowly crosslinks the PVA ${ }^{43,44}$. The resulting crosslinked network contained some bubbles, which form initially due to entrainment of air during the mixing of the PVA with borax. Efforts to remove these bubbles by degassing (by exposing the material to vacuum) resulted in even more bubbles due to evaporation of water.

An extensometer measured the mechanical properties of the cured SE 1700 and PVA (Experimental Section 3 in SI). Figure 3d plots the tensile stress as a function of tensile strain. It shows that SE 1700 sample is elastic with a modulus of $0.91 \mathrm{Mpa}$ and extends beyond $250 \%$ strain before breaking. In contrast, the crosslinked PVA is stiff and its modulus is nearly $100 \mathrm{Mpa}$. Due to the limitations of the grips, we were unable to measure the exact failure strain of the PVA, although by hand it was apparent that it failed at low strains. Nevertheless, Figure 3d clearly demonstrates that the process is capable of producing encapsulated wires with a wide range of mechanical properties.

We also measured the rheological properties of the polymers prior to crosslinking to understand what characteristics may enable the formation of wires. We reasoned that there are three important factors to enable the drawing method: 1) the chosen encasing materials should possess sufficiently high viscosity (or yield stress) so the metal does not sink or penetrate the materials during the drawing process; 2) the adhesion between the metal and the encasing materials should be sufficient to convey enough stress on the oxide-coated metal to break the oxide skin during stretching; 3 ) the oxide skin should break and reform during the elongation of metal.

Figure 3d reports the frequency spectra of the storage ( $\left.G^{\prime}\right)$ and loss $\left(G^{\prime \prime}\right)$ moduli of all samples at room temperature obtained through dynamic rheology in the linear viscoelastic regime using a rheometer (Experimental Section 4 in SI). The putty and 
aqueous PVA are classic viscoelastic materials exhibiting a frequency-dependent terminal regime at low frequencies with a moduli crossover and plateau region at higher frequencies ${ }^{45,46}$. These materials are sufficiently stiff and have enough zero-shear viscosity (Figure 3f) that they can be handled by hand, but flow readily when pulled. In contrast, the SE 1700 is a gel, with $G^{\prime}$ being larger than G' and both are relatively independent of frequency ${ }^{45-47}$. As such, this material has a yield stress, which is also evident from the absence of a zero-shear viscosity (Figure 3f). Quantitative measurement of the yield stress obtained by plotting the elastic stress ( $G^{\prime}$ times strain) as a function of $\operatorname{strain}^{47-49}$ reveals a value of approximately $300 \mathrm{~Pa} \pm 15$ Pa. The yield stress allows the gel to hold its shape prior to and after deformation, as well as support the metal. However, the yield stress is sufficiently low that it yields during handling (for this reason, we elongated this gel on a carrier substrate since it yielded readily when gripped directly by hand). Therefore, both viscoelastic polymers as well as gels with yield stress are compatible with this method.

We speculated that any liquid with a sufficiently high viscosity would be compatible with the drawing method since large viscosities retard the gravitational pull of the dense liquid metal $(6.25 \mathrm{~g} / \mathrm{mL})$ through the less dense liquid (on the time scale of the drawing process). To estimate the minimum viscosity, we utilized the equation for a sphere falling through a viscous medium (Stokes' law ${ }^{50}$ ). For the time scale of the drawing process $(\sim 10$ seconds), we estimated the minimum viscosity that would prevent the droplet of metal from sinking more than $1 / 2$ its diameter into the supporting polymer. This estimate predicts a minimum viscosity of $500 \mathrm{~Pa} \cdot \mathrm{s}$, assuming a steady state velocity. We were unable to find purely viscous fluids near this threshold, but we did verify that the metal sinks readily through fluids well below this threshold, such as water $(0.001 \mathrm{~Pa} \cdot \mathrm{s})$ and honey $(\sim 10 \mathrm{~Pa} \cdot \mathrm{s})$. We also confirmed that a 50 microliter droplet ( $\sim 2 \mathrm{~mm}$ radius) of the metal falls through the putty at a constant rate of $\sim 1.6 \mu \mathrm{m} / \mathrm{s}$ (Figure S1 in SI), which is in agreement with Stoke's law for the viscosity of the putty measured by rheology $\left(\sim 1 \times 10^{5} \mathrm{~Pa} \cdot s\right)$. This measurement helps justify the use of Stoke's law for estimating the minimum viscosity and quantifies how slowly the metal settles in the polymers utilized in this 
study.

The rheological measurements also provide an estimate of the forces involved with drawing the wires. We assume that the metal elongates when the stresses exceed the surface yield stress of the oxide on the liquid metal $(0.6 \mathrm{~N} / \mathrm{m})^{10,51}$. Thus, the metal will elongate at a force of approximately $0.6 \times D$. The diameter of the metal ranges from $1 \mathrm{~mm}$ to $10 \mu \mathrm{m}$; thus the largest force required for elongation would be $<1 \mathrm{mN}$. To prove that this force is insignificant, we estimate the force required to elongate the polymer. According to the rheological measurements, the effective modulus of the putty is in the range of $10^{4}-10^{5} \mathrm{~Pa}$ over a wide range of rates (we assume the extension modulus is $\sim 3 \mathrm{x}$ the shear modulus measured by rheology). The viscoelastic polymers start as slabs of approximately $2 \times 2 \mathrm{~cm}^{2}$ in cross section and are drawn over the course of tens of seconds. Thus, the force necessary to elongate the putty is approximately a few Newtons. This scaling analysis suggests that it is easy to elongate the slab by hand and that the forces needed to yield the metal are significantly smaller than those needed to elongate the polymer. Interestingly, the bulk forces required to elongate the polymer becomes less than the surface forces necessary to elongate the liquid metal when the length scales of both materials go below order $10 \mu \mathrm{m}$, which may explain why we did not observe wires smaller than this length scale. 
a
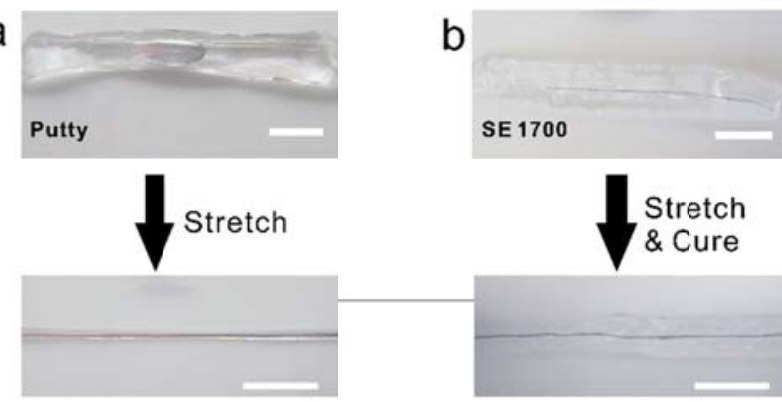

C

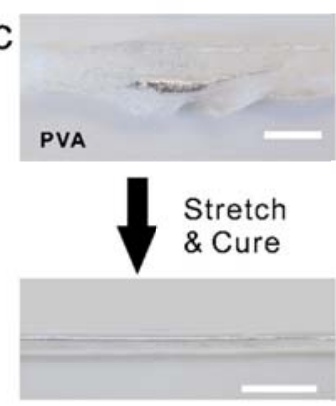

d

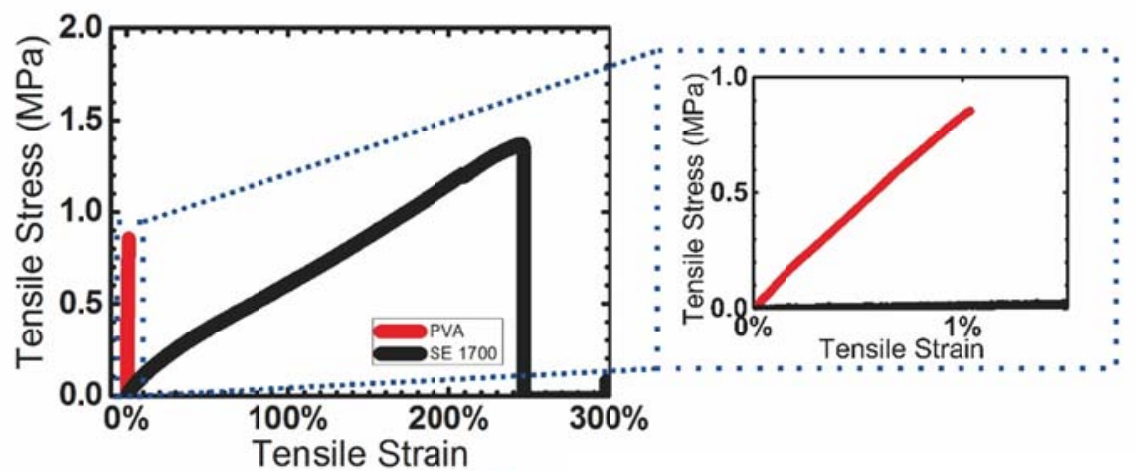

e
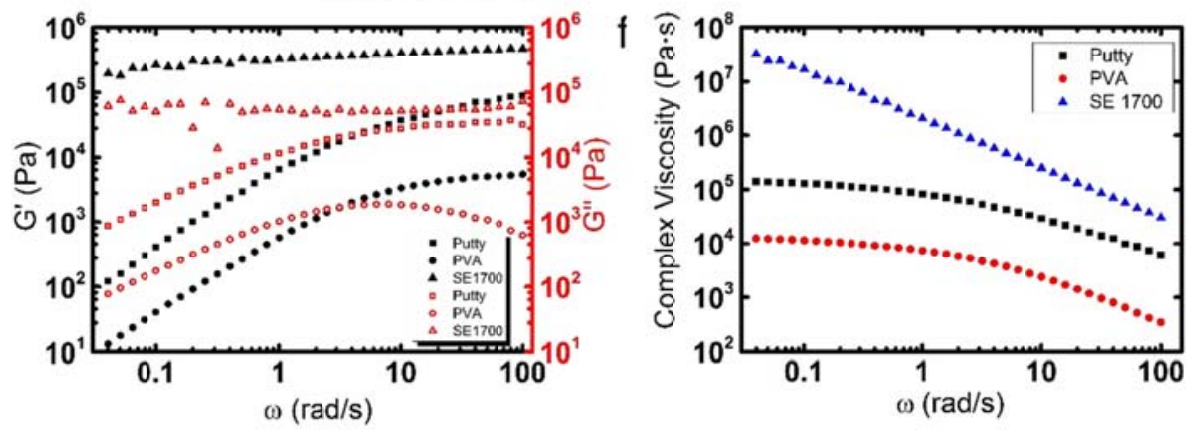

Figure 3. Fabrication of wires consisting of a metal core and insulating shell of three disparate materials (scale bar $10 \mathrm{~mm}$ ). (a) Fabrication of core-shell metal wires using putty. (b) Fabrication of core-shell metal wires using SE 1700 pre-polymer gel. (c) Fabrication of core-shell metal wires using PVA (with water and borax). (d) Plot of the tensile stress as a function of tensile strain for cured PVA and SE 1700 samples. (e) Plot of the storage modulus and loss modulus as a function of frequency for putty, PVA, SE 1700 samples before curing (G' closed symbols, G" open symbols).

Plot of complex viscosity as a function of frequency for putty, PVA, SE 1700 before curing.

Figure 3 demonstrates the possibility to fabricate single core-shell liquid metal 
wires. However, other more complex geometries are possible based on this drawing method, which provides a simple method for patterning liquid metal. The method here is capable of creating liquid metal structures smaller than most existing techniques in a much simpler fashion. There are techniques capable of patterning below 10 microns, but these exceptions typically require additional equipment or molds.

To illustrate the utility of this method for patterning liquid metal structures, we placed an EGaIn wire supported by putty across two electrodes connected to light-emitting diodes (LEDs) on a glass slide. Over the course of a few days, the wire sinks and penetrates through the insulating putty to electronically connect the circuit and light up the LEDs, as shown in Figure 4a. Equally interestingly, the insulating polymer can be removed to form a freestanding liquid metal wire. Figure 4b demonstrates that formic acid dissolves PVA after drawing an EGaIn wire. The surface oxide on the metal stabilizes the freestanding wire.
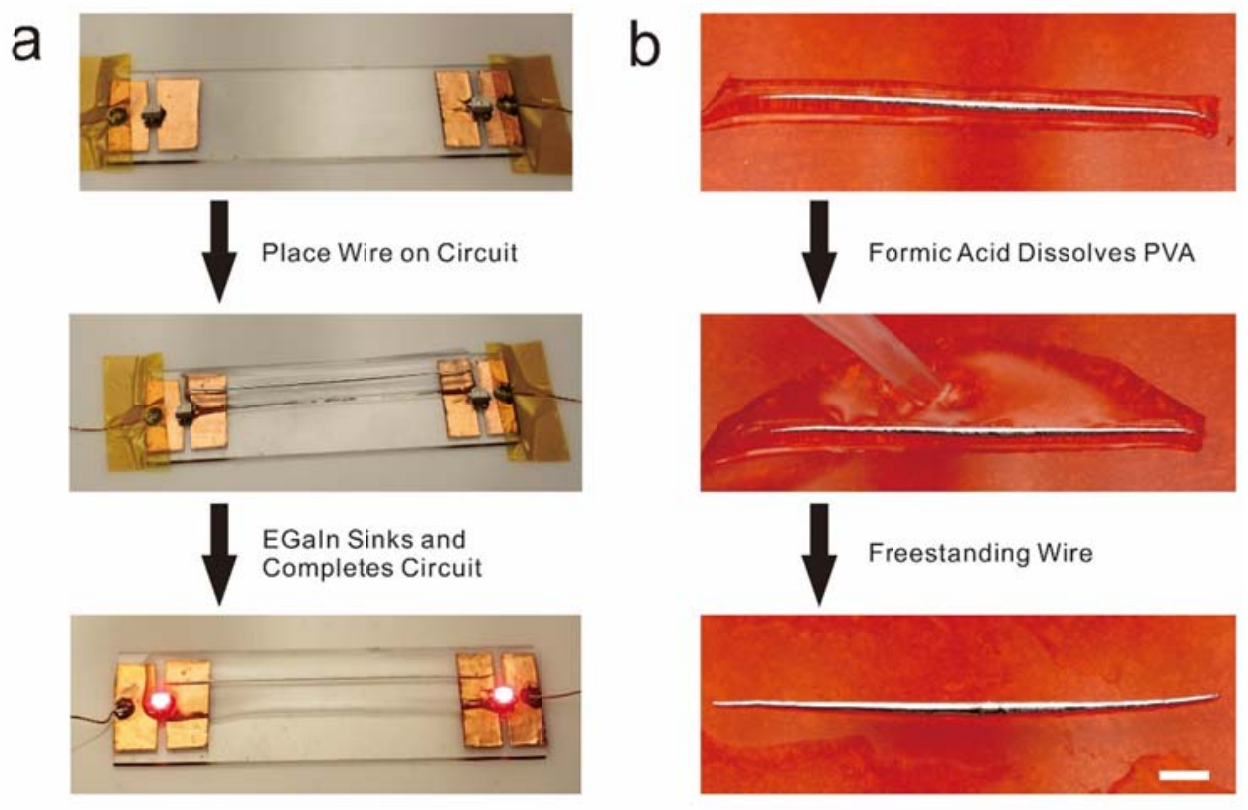

Figure 4. Patterning of wires for "wires on demand". (a) After draping an EGaIn wire in putty across a circuit, the EGaIn sinks through the putty to complete the electrical circuit. (b) Dissolving away an encapsulating polymer (PVA) produces free-standing liquid metal wires. The scale bar is $2 \mathrm{~mm}$ 
It is also possible to fabricate other complex geometries, such as parallel wires, branched wires, and helices. Stretching and overlapping the encased wires multiple times results in parallel structures. The parallel network of wires is quite homogenous, as shown in Figure 5a, since each wire has similar strain history. Figure 5b shows that stretching the polymer substrate in multiple directions results in branched liquid metal lines. In addition, a fiber can be wound into a ring and then distorted into an elongated loop structure, as shown in Figure 5c. It is also possible to create curvilinear structures by twisting two separate wires in putty. The putty merges together into a single structure containing metal lines, such as the double helix structure shown in Figure 5d. Figure 5e shows stretchable and twistable elastomeric wires (cured SE 1700). Furthermore, the wires can connect with other circuit elements, such as light-emitting diodes (LED), to complete a circuit. Figure 5f contains an image of helical wires connected to a LED. 
a

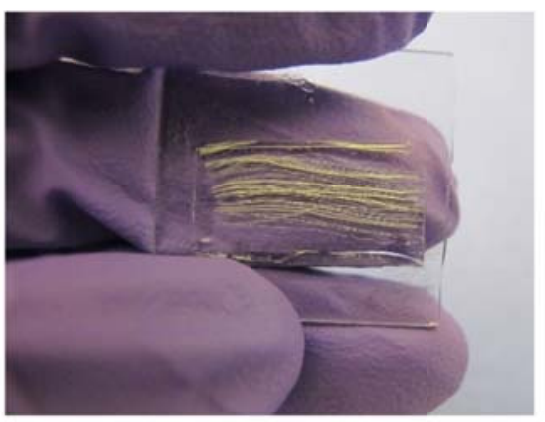

c

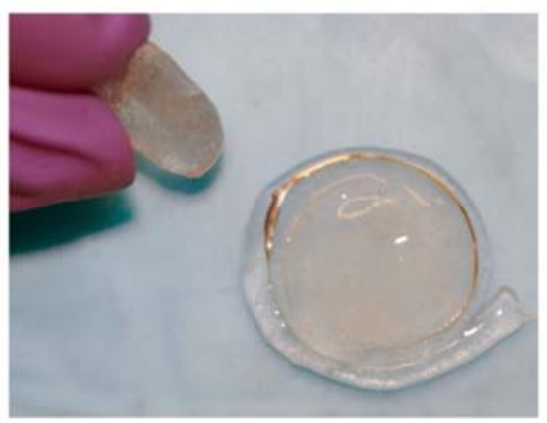

d
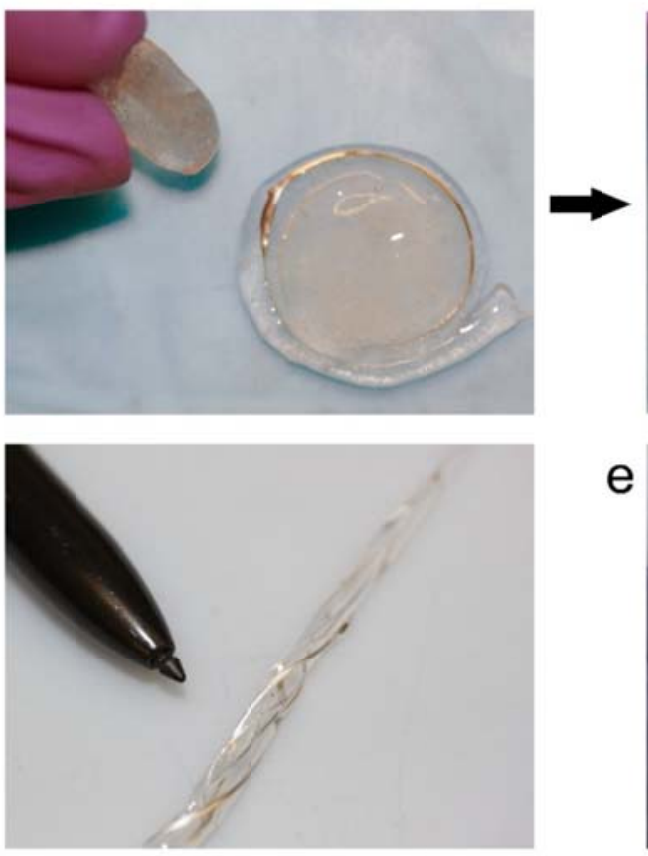

e

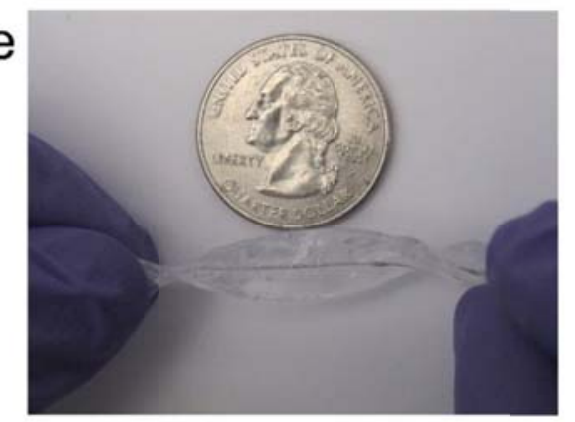

f

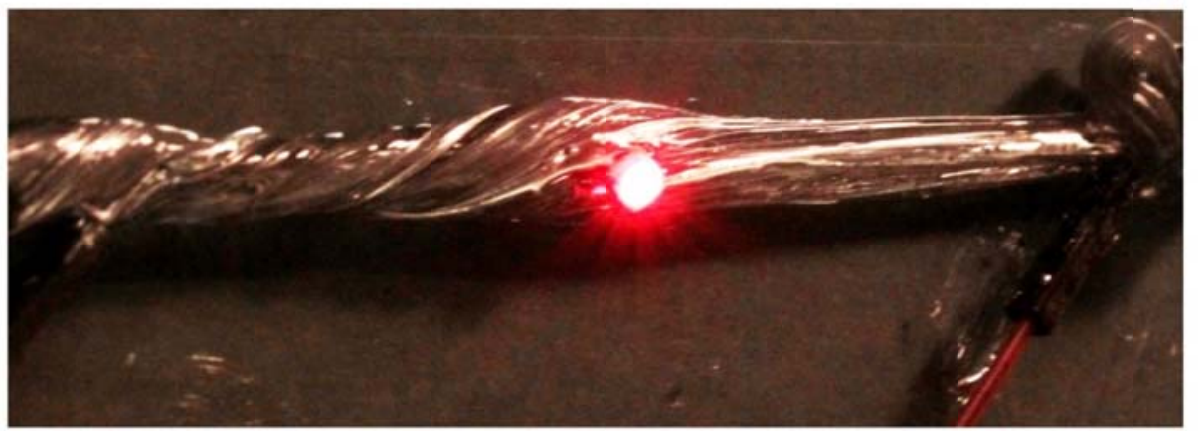

Figure 5. Metal wires with various geometries formed at room temperature.

Parallel networks of metal lines. (b) Branched wires on a PVA substrate. (c) Branched wires obtained by first contacting a wire loop with additional PVA and then stretching. (d) Helical wires. (e) Stretchable and twistable wires. (f) Helices of liquid metal wires can light up an LED inserted into the putty. 
Although the formation of wires is simple, there are some challenges to this method that arise mainly due to manual processing. For example, 1) it is challenging to control the strains precisely (and therefore necking or defects can occur at large strains or at strain rates $>1 \mathrm{~cm} / \mathrm{s}$ ); 2) processing wires with diameters below $10 \mu \mathrm{m}$ is very difficult; 3) although individual wires form within seconds, manual processing limits the throughput of the number of wires produced; and 4) drawing suspended wires at slow strain rates $(<<1 \mathrm{~cm} / \mathrm{s})$ can result in sagging of the wire due to gravity. These issues could be potentially addressed by utilizing mechanized operations or by utilizing localized heating (or crosslinking) to control the mechanical properties (and thus, the strain), as done with drawing optical fibers.

\section{Conclusion}

This paper reports an extremely facile method to draw metallic wires by hand at room temperature by stretching liquid metal on polymeric substrates composed of gels or viscoelastic polymers. The strain of the polymer substrate controls the diameter of the wires, which can narrow down to tens of microns. Moreover, geometries beyond simple wires are possible including parallel, core-shell, branched, and helix structures. The resulting wires can be stretchable, soft, or rigid depending on the polymer and this principle could extend to other encasing materials with a range of chemical and mechanical compositions. Although the ability to form structures by hand is appealing, the method would improve with automation to increase the length and uniformity of the fibers. The resulting fibers have potential applications in soft and stretchable electronics, or new applications, such as 'wires on demand' for repairing electrical connections. 


\section{Acknowledgements}

The authors acknowledge funding from the National Science Foundation through CAREER (CMMI-0954321) and the Research Triangle MRSEC (DMR-1121107). We thank Mr. Matthew Melillo for providing Teflon molds for mechanical measurements and the GEAR program for support.

\section{Author contributions}

Y.L., C.L., J.G., S.K. and M.D. designed the project. Y.L., C.L., W.S. and A.M. performed the experiments. Y.L., S.K. and M.D. wrote the paper. All authors analyzed and interpreted the data.

\section{Competing financial interests}

The authors declare no competing financial interests.

\section{References}

(1) Jaeger, R. E. Fiber Drawing Process: Characterization and Control. In Fiber Optics; Bendow, B.; Mitra, S. S., Eds.; Springer US, 1979; pp. 33-53.

(2) Hou, C.; Jia, X.; Wei, L.; Tan, S.-C.; Zhao, X.; Joannopoulos, J. D.; Fink, Y. Crystalline Silicon Core Fibres from Aluminium Core Preforms. Nat. Commun. 2015, 6, 6248 .

(3) Abouraddy, A. F.; Bayindir, M.; Benoit, G.; Hart, S. D.; Kuriki, K.; Orf, N.; Shapira, O.; Sorin, F.; Temelkuran, B.; Fink, Y. Towards Multimaterial Multifunctional Fibres That See, Hear, Sense and Communicate. Nat. Mater. 2007, 6, 336-347.

(4) Yaman, M.; Khudiyev, T.; Ozgur, E.; Kanik, M.; Aktas, O.; Ozgur, E. O.; Deniz, H.; Korkut, E.; Bayindir, M. Arrays of Indefinitely Long Uniform Nanowires and Nanotubes. Nat. Mater. 2011, 10, 494-501.

(5) Canales, A.; Jia, X.; Froriep, U. P.; Koppes, R. A.; Tringides, C. M.; Selvidge, J.; Lu, C.; Hou, C.; Wei, L.; Fink, Y.; et al. Multifunctional Fibers for Simultaneous Optical, Electrical and Chemical Interrogation of Neural Circuits in Vivo. Nat. Biotechnol. 2015, 33, 277-284.

(6) Wistreich, J. G. The Fundamentals of Wire Drawing. Metall. Rev. 1958, 3, 97 142.

(7) Zhu, S.; So, J.-H.; Mays, R.; Desai, S.; Barnes, W. R.; Pourdeyhimi, B.; Dickey, M. D. Ultrastretchable Fibers with Metallic Conductivity Using a Liquid Metal Alloy Core. Adv. Funct. Mater. 2013, 23, 2308-2314.

(8) Mineart, K. P.; Lin, Y.; Desai, S. C.; Krishnan, A. S.; Spontak, R. J.; Dickey, M. 
D. Ultrastretchable, Cyclable and Recyclable 1- and 2-Dimensional Conductors Based on Physically Cross-Linked Thermoplastic Elastomer Gels. Soft Matter 2013, 9, 7695.

(9) French, S. J.; Saunders, D. J.; Ingle, G. W. The System Gallium-Indium. J. Phys. Chem. 1938, 42, 265-274.

(10) Dickey, M. D.; Chiechi, R. C.; Larsen, R. J.; Weiss, E. A.; Weitz, D. A.; Whitesides, G. M. Eutectic Gallium-Indium (EGaIn): A Liquid Metal Alloy for the Formation of Stable Structures in Microchannels at Room Temperature. Adv. Funct. Mater. 2008, 18, 1097-1104.

(11) Lu, Y.; Hu, Q.; Lin, Y.; Pacardo, D. B.; Wang, C.; Sun, W.; Ligler, F. S.; Dickey, M. D.; Gu, Z. Transformable Liquid-Metal Nanomedicine. Nat. Commun. 2015, 6, 10066.

(12) Zrnic, D.; Swatik, D. S. On the Resistivity and Surface Tension of the Eutectic Alloy of Gallium and Indium. J. Common Met. 1969, 18, 67-68.

(13) Dickey, M. D. Emerging Applications of Liquid Metals Featuring Surface Oxides. ACS Appl. Mater. Interfaces 2014, 6, 18369-18379.

(14) Koo, H.-J.; So, J.-H.; Dickey, M. D.; Velev, O. D. Towards All-Soft Matter Circuits: Prototypes of Quasi-Liquid Devices with Memristor Characteristics. Adv. Mater. 2011, 23, 3559-3564.

(15) So, J.-H.; Thelen, J.; Qusba, A.; Hayes, G. J.; Lazzi, G.; Dickey, M. D. Reversibly Deformable and Mechanically Tunable Fluidic Antennas. Adv. Funct. Mater. 2009, 19, 3632-3637.

(16) Rashed Khan, M.; Hayes, G. J.; So, J.-H.; Lazzi, G.; Dickey, M. D. A Frequency Shifting Liquid Metal Antenna with Pressure Responsiveness. Appl. Phys. Lett. 2011, 99, 013501.

(17) Cheng, S.; Rydberg, A.; Hjort, K.; Wu, Z. Liquid Metal Stretchable Unbalanced Loop Antenna. Appl. Phys. Lett. 2009, 94, 144103.

(18) Morishita, A. M.; Kitamura, C. K. Y.; Ohta, A. T.; Shiroma, W. A. A Liquid-Metal Monopole Array With Tunable Frequency, Gain, and Beam Steering. IEEE Antennas Wirel. Propag. Lett. 2013, 12, 1388-1391.

(19) Cheng, S.; Wu, Z.; Hallbjorner, P.; Hjort, K.; Rydberg, A. Foldable and Stretchable Liquid Metal Planar Inverted Cone Antenna. IEEE Trans. Antennas Propag. 2009, 57, 3765-3771.

(20) Chiechi, R. C.; Weiss, E. A.; Dickey, M. D.; Whitesides, G. M. Eutectic Gallium-Indium (EGaIn): A Moldable Liquid Metal for Electrical Characterization of Self-Assembled Monolayers. Angew. Chem. 2008, 120, 148-150.

(21) So, J.-H.; Dickey, M. D. Inherently Aligned Microfluidic Electrodes Composed of Liquid Metal. Lab. Chip 2011, 11, 905.

(22) Tang, S.-Y.; Khoshmanesh, K.; Sivan, V.; Petersen, P.; O’Mullane, A. P.; Abbott, D.; Mitchell, A.; Kalantar-zadeh, K. Liquid Metal Enabled Pump. Proc. Natl. Acad. Sci. 2014, 111, 3304-3309.

(23) Fassler, A.; Majidi, C. Soft-Matter Capacitors and Inductors for Hyperelastic Strain Sensing and Stretchable Electronics. Smart Mater. Struct. 2013, 22, 
055023.

(24) Liu, S.; Sun, X.; Hildreth, O. J.; Rykaczewski, K. Design and Characterization of a Single Channel Two-Liquid Capacitor and Its Application to Hyperelastic Strain Sensing. Lab. Chip 2015, 15, 1376-1384.

(25) Lin, Y.; Cooper, C.; Wang, M.; Adams, J. J.; Genzer, J.; Dickey, M. D. Handwritten, Soft Circuit Boards and Antennas Using Liquid Metal Nanoparticles. Small 2015, 11, 6397-6403.

(26) Park, Y.-L.; Majidi, C.; Kramer, R.; Bérard, P.; Wood, R. J. Hyperelastic Pressure Sensing with a Liquid-Embedded Elastomer. J. Micromechanics Microengineering 2010, 20, 125029.

(27) Palleau, E.; Reece, S.; Desai, S. C.; Smith, M. E.; Dickey, M. D. Self-Healing Stretchable Wires for Reconfigurable Circuit Wiring and 3D Microfluidics. Adv. Mater. 2013, 25, 1589-1592.

(28) Blaiszik, B. J.; Jones, A. R.; Sottos, N. R.; White, S. R. Microencapsulation of Gallium-indium (Ga-In) Liquid Metal for Self-Healing Applications. J. Microencapsul. 2014, 31, 350-354.

(29) Kramer, R. K.; Majidi, C.; Sahai, R.; Wood, R. J. Soft Curvature Sensors for Joint Angle Proprioception. In 2011 IEEE/RSJ International Conference on Intelligent Robots and Systems (IROS); 2011; pp. 1919-1926.

(30) Kim, H.-J.; Son, C.; Ziaie, B. A Multiaxial Stretchable Interconnect Using Liquid-Alloy-Filled Elastomeric Microchannels. Appl. Phys. Lett. 2008, 92, 011904.

(31) Park, J.; Wang, S.; Li, M.; Ahn, C.; Hyun, J. K.; Kim, D. S.; Kim, D. K.; Rogers, J. A.; Huang, Y.; Jeon, S. Three-Dimensional Nanonetworks for Giant Stretchability in Dielectrics and Conductors. Nat. Commun. 2012, 3, 916.

(32) Joshipura, I. D.; Ayers, H. R.; Majidi, C.; Dickey, M. D. Methods to Pattern Liquid Metals. J Mater Chem C 2015, 3, 3834-3841.

(33) Jeong, S. H.; Hagman, A.; Hjort, K.; Jobs, M.; Sundqvist, J.; Wu, Z. Liquid Alloy Printing of Microfluidic Stretchable Electronics. Lab. Chip 2012, 12, 4657-4664.

(34) Kramer, R. K.; Majidi, C.; Wood, R. J. Masked Deposition of Gallium-Indium Alloys for Liquid-Embedded Elastomer Conductors. Adv. Funct. Mater. 2013, 23, 5292-5296.

(35) Gozen, B. A.; Tabatabai, A.; Ozdoganlar, O. B.; Majidi, C. High-Density Soft-Matter Electronics with Micron-Scale Line Width. Adv. Mater. 2014, 26, 5211-5216.

(36) Tabatabai, A.; Fassler, A.; Usiak, C.; Majidi, C. Liquid-Phase Gallium-Indium Alloy Electronics with Microcontact Printing. Langmuir 2013, 29, 6194-6200.

(37) Ladd, C.; So, J.-H.; Muth, J.; Dickey, M. D. 3D Printing of Free Standing Liquid Metal Microstructures. Adv. Mater. 2013, 25, 5081-5085.

(38) Yang, H.; Lightner, C. R.; Dong, L. Light-Emitting Coaxial Nanofibers. ACS Nano 2012, 6, 622-628.

(39) Castano, L. M.; Flatau, A. B. Smart Fabric Sensors and E-Textile Technologies: A Review. Smart Mater. Struct. 2014, 23, 053001. 
(40) Dubas, S. T.; Kumlangdudsana, P.; Potiyaraj, P. Layer-by-Layer Deposition of Antimicrobial Silver Nanoparticles on Textile Fibers. Colloids Surf. Physicochem. Eng. Asp. 2006, 289, 105-109.

(41) Dalton, A. B.; Collins, S.; Muñoz, E.; Razal, J. M.; Ebron, V. H.; Ferraris, J. P.; Coleman, J. N.; Kim, B. G.; Baughman, R. H. Super-Tough Carbon-Nanotube Fibres. Nature 2003, 423, 703-703.

(42) Doudrick, K.; Liu, S.; Mutunga, E. M.; Klein, K. L.; Damle, V.; Varanasi, K. K.; Rykaczewski, K. Different Shades of Oxide: From Nanoscale Wetting Mechanisms to Contact Printing of Gallium-Based Liquid Metals. Langmuir 2014, 30, 6867-6877.

(43) Chen, C. Y.; Yu, T.-L. Dynamic Light Scattering of Poly(vinyl Alcohol)-Borax Aqueous Solution near Overlap Concentration. Polymer 1997, 38, 2019-2025.

(44) Inoue, T.; Osaki, K. Rheological Properties of Poly(vinyl Alcohol)/sodium Borate Aqueous Solutions. Rheol. Acta 1993, 32, 550-555.

(45) Raghavan, S. R.; Hou, J.; Baker, G. L.; Khan, S. A. Colloidal Interactions between Particles with Tethered Nonpolar Chains Dispersed in Polar Media:

Direct Correlation between Dynamic Rheology and Interaction Parameters. Langmuir 2000, 16, 1066-1077.

(46) Raghavan, S. R.; Walls, H. J.; Khan, S. A. Rheology of Silica Dispersions in Organic Liquids: New Evidence for Solvation Forces Dictated by Hydrogen Bonding. Langmuir 2000, 16, 7920-7930.

(47) Burns, N. A.; Naclerio, M. A.; Khan, S. A.; Shojaei, A.; Raghavan, S. R. Nanodiamond Gels in Nonpolar Media: Colloidal and Rheological Properties. $J$. Rheol. 1978-Present 2014, 58, 1599-1614.

(48) Walls, H. J.; Caines, S. B.; Sanchez, A. M.; Khan, S. A. Yield Stress and Wall Slip Phenomena in Colloidal Silica Gels. J. Rheol. 1978-Present 2003, 47, 847-868.

(49) Yang, M.-C.; Scriven, L. E.; Macosko, C. W. Some Rheological Measurements on Magnetic Iron Oxide Suspensions in Silicone Oil. J. Rheol. 1978-Present 1986, 30, 1015-1029.

(50) Batchelor, G. K. An Introduction to Fluid Dynamics; Cambridge University Press: Cambridge, 2000.

(51) Larsen, R. J.; Dickey, M. D.; Whitesides, G. M.; Weitz, D. A. Viscoelastic Properties of Oxide-Coated Liquid Metals. J. Rheol. 1978-Present 2009, 53, 1305-1326. 